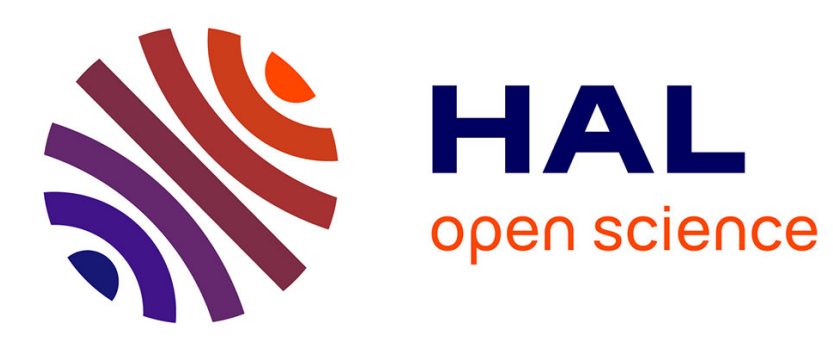

\title{
La diffusion des NTIC au sein du réseau urbain français et le rôle des acteurs publics
}

Pascal Gillon, Oumar N’Diaye, Patrice Caro

\section{To cite this version:}

Pascal Gillon, Oumar N'Diaye, Patrice Caro. La diffusion des NTIC au sein du réseau urbain français et le rôle des acteurs publics. NETCOM: Réseaux, communication et territoires / Networks and Communications Studies, 2001, 15 (3-4), pp.231-256. halshs-00840489

\section{HAL Id: halshs-00840489 \\ https://shs.hal.science/halshs-00840489}

Submitted on 11 Jul 2013

HAL is a multi-disciplinary open access archive for the deposit and dissemination of scientific research documents, whether they are published or not. The documents may come from teaching and research institutions in France or abroad, or from public or private research centers.
L'archive ouverte pluridisciplinaire HAL, est destinée au dépôt et à la diffusion de documents scientifiques de niveau recherche, publiés ou non, émanant des établissements d'enseignement et de recherche français ou étrangers, des laboratoires publics ou privés. 


\title{
La diffusion des NTIC au sein du réseau urbain français et le rôle des acteurs publics
}

\author{
Pascal Gillon', Oumar N'Diaye ${ }^{2}$, Patrice Caro ${ }^{3}$
}

\begin{abstract}
Résumé.- L'étude porte sur l'utilisation des NTIC par le grand public et les acteurs publics. D'une part, nous analysons la localisation des sites Internet en France à partir des données fournies par l'AFNIC, organisme centralisateur des noms de domaine en ".fr". À partir de cet échantillon (un quart des sites français), nous retrouvons une partition assez nette de l'espace français, entre la région parisienne, les métropoles d'équilibre et le reste de la France. D'autre part, nous tentons de recenser les initiatives menées par les institutions publiques en charge de l'aménagement du territoire dans le domaine des NTIC. Après avoir explicité le cadre réglementaire et économique dans lequel les collectivités sont obligées de travailler, nous décrivons les principales initiatives en tentant d'expliciter les motivations des partenaires. La partition de l'espace est dans ce cas beaucoup moins nette, puisque les acteurs publics en milieu rural ont parfois une conscience plus aiguë des possibilités qu'offrent les NTIC que les acteurs publics en milieu urbain.
\end{abstract}

Mots-clés.- NTIC, Sites Internet, Fournisseurs d'accès Internet, Localisation, Diffusion, Hiérarchie urbaine, Acteurs publics

Abstract.- The study deals with the ways in which the general public and the government make use of new information and communication technologies. We analyze the physical locations of Internet sites with "fr" as their domain names. The data were provided by the French NIC center (AFNIC). Using this sample (a quarter of all French sites), we find a rather even distribution of French sites in the Paris area plus the other main French cities, and the remainder of France. In addition, we try to enumerate the initiatives of the public actors responsible for the regional planning of the new information and communication technologies. Firstly, we clarify the economic and legal framework in which public actors are obliged to work. Then, we describe the principal projects and the main motivations of the partners involved. In this case, the distribution is much less clear, because rural actors are sometimes more conscious than urban actors about the possibilities offered by new information and communication technologies.

Key words: ICT, Web sites, Web providers localization, Diffusion, Central place theory, Public actors

1. Dr Pascal Gillon, Laboratoire THEMA/CNRS, Maître de Conférence de Géographie à l'Université de Franche-Comté, 25030 Besançon, France. E-Mail : pascal.gillon@univ-fcomte.fr.

2. Dr Oumar Ndiaye, Chargé d'études en NTIC au Laboratoire THEMA/CNRS, Université de Franche-Comté, 25030 Besançon, France. E-Mail : oumar.ndiaye@univ-fcomte.fr.

3. Dr Patrice Caro, Laboratoire THEMA/CNRS, Maître de Conférence de Géographie à l'Université de Franche-Comté, 25030 Besançon, France. E-Mail : patrice.caro@univ-fcomte.fr. 


\section{INTRODUCTION}

Le terme NTIC (Nouvelles Technologies de l'Information et de la Communication) désigne l'ensemble des technologies liées aux transmissions de données sous forme numérique. Les NTIC se sont largement diffusées en France au début des années 1990 (Internet: croissance à partir de 1996). Il existe à l'heure actuelle peu de travaux identifiant l'état de la diffusion de ces nouveautés sur le territoire national. Concernant l'utilisation d'Internet, L. Grasland $(1997,1998)$ a montré que parallèlement au mécanisme habituel de diffusion d'innovation technique ou industrielle, où les villes sont privilégiées, des initiatives sont apparues précocement dans le milieu rural.

Les nouvelles technologies recouvrent plusieurs champs au sein desquels l'utilisation d'Internet, et en amont la mise en place des réseaux physiques permettant cette utilisation, constituent une sorte de noyau dur. Nous nous proposons d'étudier la diffusion des NTIC au sein de l'espace français. Le réseau urbain concentre-t-il l'essentiel des utilisateurs et des réseaux physiques? Si tel est le cas, quels sont les facteurs déterminant cette diffusion?

Bien que les données relatives à cette question soient difficilement accessibles, comparables et spatialisables, pour certaines d'entre elles, nous présentons ici quelques indicateurs relatifs à l'utilisation d'Internet (fournisseurs d'accès à Internet FAI- ou provider, sites en ligne ".fr"). Toutefois, il ne s'agit que d'une photographie datée de la fin de l'année 1998. Les réseaux et le nombre d'utilisateurs se sont encore développés depuis. Par ailleurs, pour donner un aperçu de l'état de développement des réseaux physiques, il faut absolument souligner le contexte dans lequel ils se développent. La libéralisation du marché des télécommunications, l'intervention de l'État au travers de la législation et la nouvelle approche institutionnelle de l'aménagement du territoire influencent profondément le développement des infrastructures. En effet, les débats actuels en matière d'aménagement du territoire (lois Chevènement et Voynet) soulignent un enjeu opposant les collectivités territoriales au pouvoir central. On ne peut faire l'économie de traiter des changements récents dans le marché des télécommunications pour mieux éclairer ces débats et ces enjeux.

Dans un premier temps, après avoir abordé la diffusion des NTIC, la localisation des utilisateurs, il sera nécessaire de raisonner par type d'infrastructures et grands domaines d'utilisation pour affiner la description de l'ancrage des NTIC sur le territoire. Dans un second temps on s'intéressera au rôle des acteurs publics. Enfin, il s'agira de présenter le contexte institutionnel et législatif dans lequel s'opère ce développement.

\section{LA DIFFUSION DES NTIC}

La France présente une situation quelque peu paradoxale en ce qui concerne l'utilisation des NTIC. En effet, nos concitoyens ont été sensibilisés très tôt à ces techniques, notamment à travers la diffusion du Minitel lancé par France Telecom. Ils se sont familiarisés avec tout d'abord la consultation de l'annuaire, 
mais aussi très rapidement avec les systèmes de réservations, le téléachat, les boîtes aux lettres électroniques, les discussions sur un réseau. Ce succès explique parmi d'autres raisons que l'usage d'Internet soit arrivé plus tard et qu'à l'heure actuelle sa diffusion n'ait pas atteint le même stade que dans d'autres pays européens (Bratzel, Dienel, 1999).

L'autre raison est le retard de l'accès à l'informatique, qui est encore faiblement développée en France, le prix de vente des ordinateurs ayant longtemps été relativement élevé. D'après une étude réalisée par l'institut de sondage allemand GfK à la fin de 1998, environ 22,5\% des familles françaises seraient équipées d'un ordinateur. Ce marché est en pleine croissance (+ $21 \%$ par rapport à 1997) puisqu'en 1996 seulement 14,5\% des ménages possédaient un appareil. Cette année, pour la première fois, il s'est vendu plus d'ordinateurs que de téléviseurs.

La mesure de l'accès à Internet aussi bien par le nombre de personnes connectées que par celui des services proposés, est assez délicate. Ce qui explique la forte variation des chiffres dans la presse nationale et spécialisée.

Notons que l'étude Médiangle, publiée le 19 mai 1999, avance un taux de 11,4 \% de Français connectés sur le web. Le même jour, Médiamétrie annonçait dans le Figaro un taux de connexion pour le trimestre de 10,4\%. Il y aurait, début 1999, environ 3,7 millions d'internautes, selon les estimations de Médiamétrie, soit un peu plus de $6 \%$ de la population française. Sont considérés comme internautes toutes les personnes, s'étant connectées à Internet ou à un service en ligne au moins une fois au cours des douze derniers mois.

La croissance actuelle des ventes d'ordinateurs laisse à penser que la France entre dans la phase la plus intense de la croissance suivant une fonction logistique. De plus, de nouvelles initiatives, comme la vente d'ordinateurs à 2000 francs contre l'engagement de prendre un abonnement sur Internet pendant deux ans, apparaissent (Société Cibox), ou encore comme la gratuité de l'accès à Internet chez de grands distributeurs d'électroménager (société Libertysurf).

D’après plusieurs sondages datant de 1998 (Consodata enquête sur 15000 personnes, Stratégies Télécom et multimédia) il est possible d'esquisser le profil de l'utilisateur type en France, assez semblable aux autres internautes.

L'utilisateur est plutôt un homme (72\%), jeune (30 ans en moyenne), percevant des revenus supérieurs à la moyenne, diplômé de l'enseignement supérieur et appartenant à une des catégories sociales et professionnelles suivantes: cadres, enseignants, chefs d'entreprise, professions libérales. On note aussi la présence relativement forte d'étudiants parmi les internautes. Cet utilisateur type habite en ville, et $25 \%$ dans une ville de plus de 100000 habitants, Paris et l'Île-de-France étant surreprésentées. Concrètement, il utilise Internet, au moins une fois par semaine, principalement pour échanger du courrier (e-mail), récupérer des informations prioritairement liées à l'actualité. Un Français sur deux utilisant Internet déclare avoir déjà acheté un produit ou service après une recherche effectuée sur le web, mais seulement un cinquième dit avoir effectué un achat en ligne. 
Après avoir brossé ce rapide portrait de l'utilisateur urbain, il est impératif d'examiner les rapports entre la localisation des fournisseurs d'accès à Internet (FAI), celle des utilisateurs et la hiérarchie urbaine.

\section{Une logique de diffusion reposant sur la hiérarchie urbaine}

L'analyse de la diffusion des sites et des fournisseurs d'accès à Internet (FAI) repose sur des données collectées par l'AFNIC'4. Celles relatives aux FAI sont facilement accessibles, puisque l'État impose leur agrément par l'AFNIC. Quant au dénombrement des sites web, il est plus délicat car l'AFNIC ne recense que les sites déclarés en ".fr", les domaines ".com", “.org" ou ".net" étant gérés par l'Internic. Nous ne traiterons donc que des sites en ".fr" au sens strict (c'est-à-dire par exemple: ne sont pas comptabilisés les sites "tm.fr", "asso.fr" et "com.fr"), compte tenu de la difficulté à mesurer les autres domaines.

\section{Les fournisseurs d'accès : une localisation uniquement urbaine}

La localisation des fournisseurs d'accès ne présente pas de contraintes spatiales: quel que soit l'endroit où ils se situent et quel que soit l'endroit d'où le client appelle, le coût de la communication est équivalent au coût d'une communication locale.

Toutefois, les 519 fournisseurs répertoriés se concentrent en quelques lieux seulement (figure 1). En effet, l'Île-de-France regroupe près d'un fournisseur sur deux (46\% du total). Paris intra-muros accueille un cinquième de l'ensemble (106 FAI). Hors de cette région, les départements abritant les neuf plus grandes agglomérations (zones d'attraction urbaine, ZAU) hébergent $22 \%$ des fournisseurs (117). Dans ces deux ensembles, représentant le sommet de la hiérarchie urbaine française, se trouvent rassemblés les trois quarts des fournisseurs d'accès pour seulement $43 \%$ de la population urbaine. À l'opposé, vingt-trois départements français, soit $12 \%$ de la population française, ne concentrent aucun fournisseur d'accès. Tous, sont des départements ruraux appartenant à la diagonale du vide, qui va des Ardennes aux Landes en passant par la Bourgogne et le sud du Massif central.

En pondérant ces mesures par la population des départements, estimée par l'INSEE pour 1995, on retrouve la même structure (figure 2). Paris et les Hauts-deSeine ont un fournisseur d'accès pour moins de 50000 habitants, alors que la moyenne nationale se situerait à un pour 110 000. Le centre Ouest français, l'Aquitaine, le littoral méditerranéen, la région Rhône-Alpes et l'Alsace se démarquent par des taux supérieurs à la moyenne.

4. AFNIC: Association française pour le nomage internet en coopération. Organisme, l'AFNIC impose un cahier des charges techniques aux fournisseurs pour l'obtention de leur agrément. Ils doivent déclarer les sites web qu'ils hébergent, avec l'adresse IP de la machine, les fournisseurs d'accès pouvant héberger aussi les pages web de leurs clients qui n'ont pas les capacités techniques ou financières pour le faire. L'AFNIC attribue alors le nom du site mais uniquement pour le domaine ".fr". 

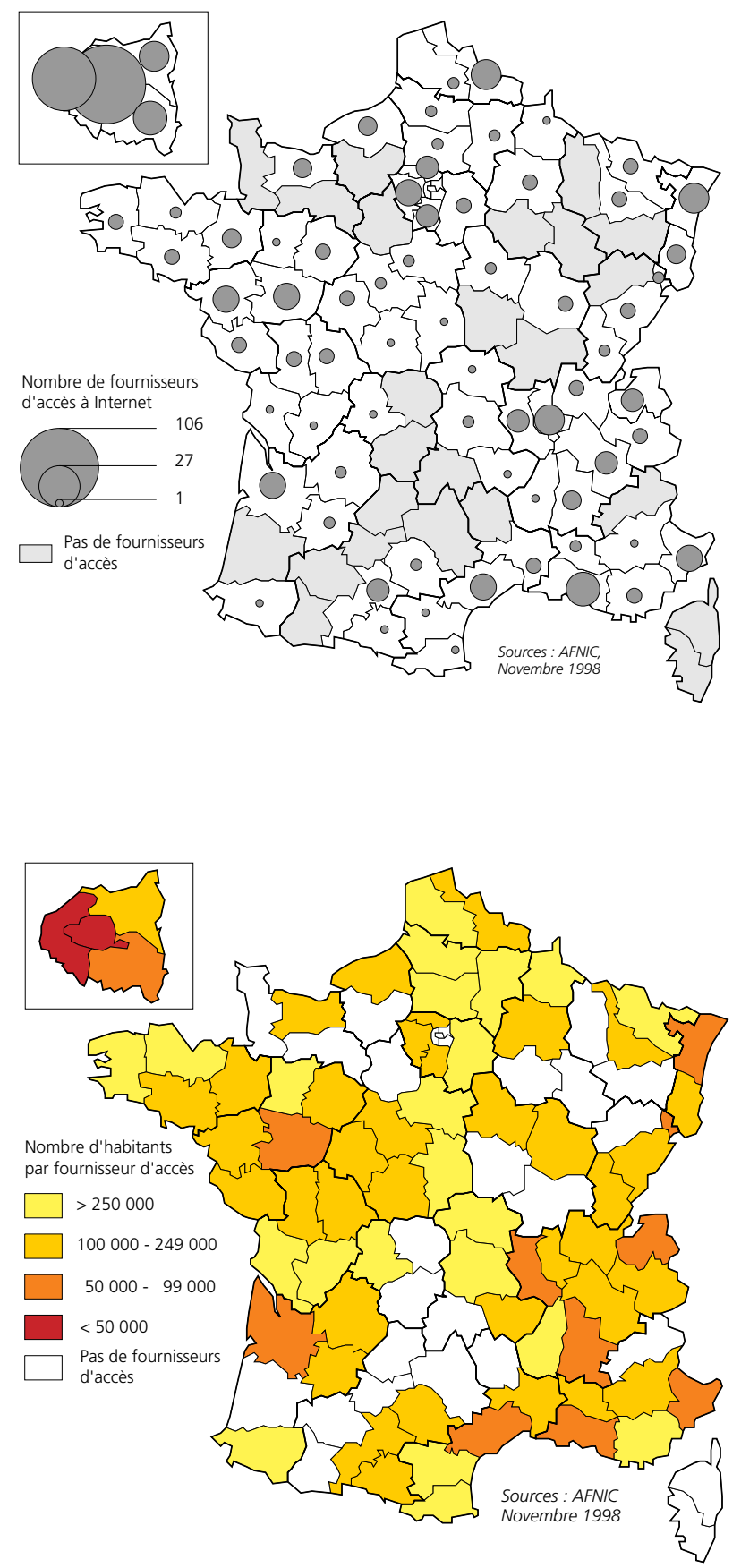

Figure 1.-Répartition

des fournisseurs d'accès

à Internet

en novembre 1998.
Figure 2.- Nombre d'habitants par fournisseur d'accès à Internet. 
À l'intérieur de chaque département, la localisation des fournisseurs d'accès est toujours l'apanage d'une ZAU. En somme, la répartition des "providers" est à l'heure actuelle indissociable du milieu urbain.

\section{Une surreprésentation des sites ".fr" en milieu urbain}

Fin 1998, des statistiques font état de l'existence d'environ 100000 sites Internet en France, dont 25000 sites en ".fr", et 25000 sites pour chaque suffixe ".com", ".org" et ".net". Là encore, nous sommes en période de croissance très rapide puisque, au 15 février 1999, les sites ".fr" sont passés au nombre de 30400.

La tendance observée pour la localisation des fournisseurs est confirmée pour les 25276 sites Internet. À savoir, la prédominance de l'Île-de-France, qui cumule $44 \%$ des sites, l'importance de Paris intra-muros qui regroupe, à lui seul, $20 \%$ du total; et les départements possédant les neuf plus grandes ZAU du sommet de la hiérarchie urbaine qui hébergent 5666 sites soit $22 \%$.

En province, les soixante-cinq départements possédant le plus petit nombre de sites Internet ne rassemblent que $10 \%$ du total de ces sites pour $41 \%$ de la population! (figure 3).

En pondérant ces mesures par le nombre d'habitants, les départements les plus urbanisés recueillent les plus forts taux de domiciliation des sites (figure 4). Paris, les Hauts-de-Seine et le Rhône possèdent en effet plus de sept sites Internet ".fr" pour 10000 habitants. Viennent ensuite certains autres départements peuplés et dynamiques comme ceux de la quasi-totalité de l'Île-de-France, de l'Alsace, du Nord, du Tarn-et-Garonne, des Alpes-Maritimes et trois départements de la région Rhône-Alpes. Les départements les plus ruraux ont un nombre de sites inférieur à deux pour 10000 habitants. La taille des départements, leur degré d'urbanisation et leur dynamisme en matière d'activités tertiaires et industrielles expliquent cette forte concentration des sites sur le territoire français. Paris et les Hauts-de-Seine par exemple constituent les plus fortes concentrations de sièges sociaux en France, voire en Europe (Saint-Julien, Caro, 1996).

En utilisant les codes postaux fournis avec l'adresse des sites, il est possible de les localiser plus précisément. Ainsi 17918 sites sont situés dans les zones d'attraction urbaines (ZAU) françaises, soit $71 \%$ du total. À l'intérieur de ces ZAU, $84,2 \%$ des sites sont localisés dans un pôle urbain et les 15,8 \% restant sont situés dans des communes périurbaines. La centralité joue donc un rôle important.

Les nombreuses initiatives prises par de petites communes rurales isolées en matière de NTIC ces dernières années n'occultent pas le fait qu'Internet demeure bien un phénomène presque purement urbain depuis sa naissance. Les fournisseurs d'accès, les utilisateurs d'Internet, mais aussi la plupart des sites ".fr", se répartissent dans les départements les plus urbanisés, voire métropolisés et à fort potentiel économique.

Il est ensuite intéressant de tester la liaison statistique qui pourrait exister entre la taille des ZAU (qui traduit de façon grossière la hiérarchie urbaine) et le 


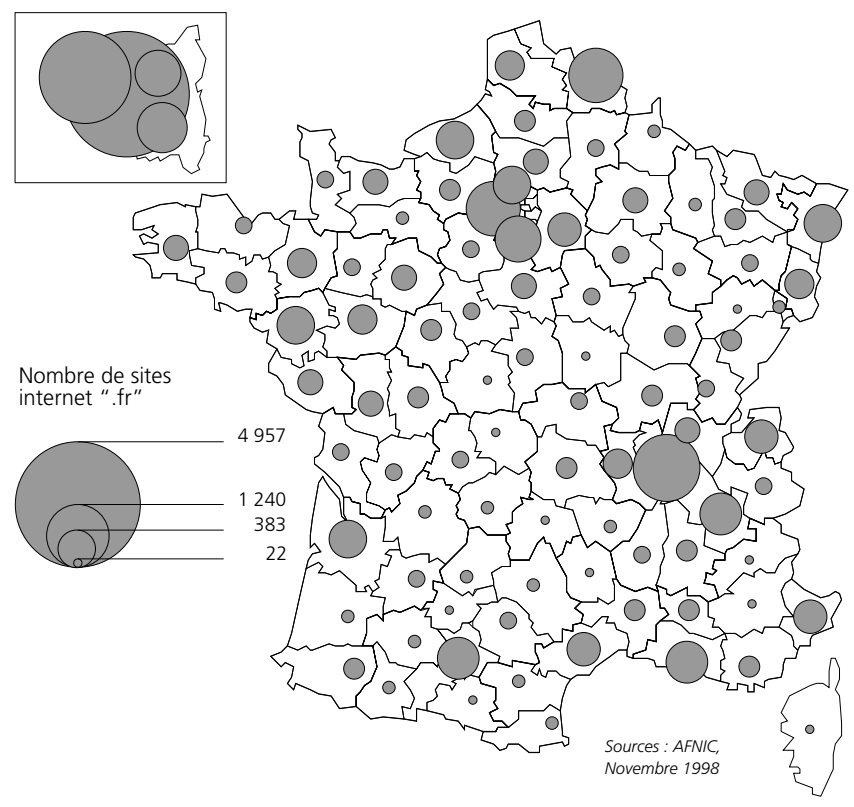

Figure 3.- Nombre de sites Internet "fr" en novembre 1998.

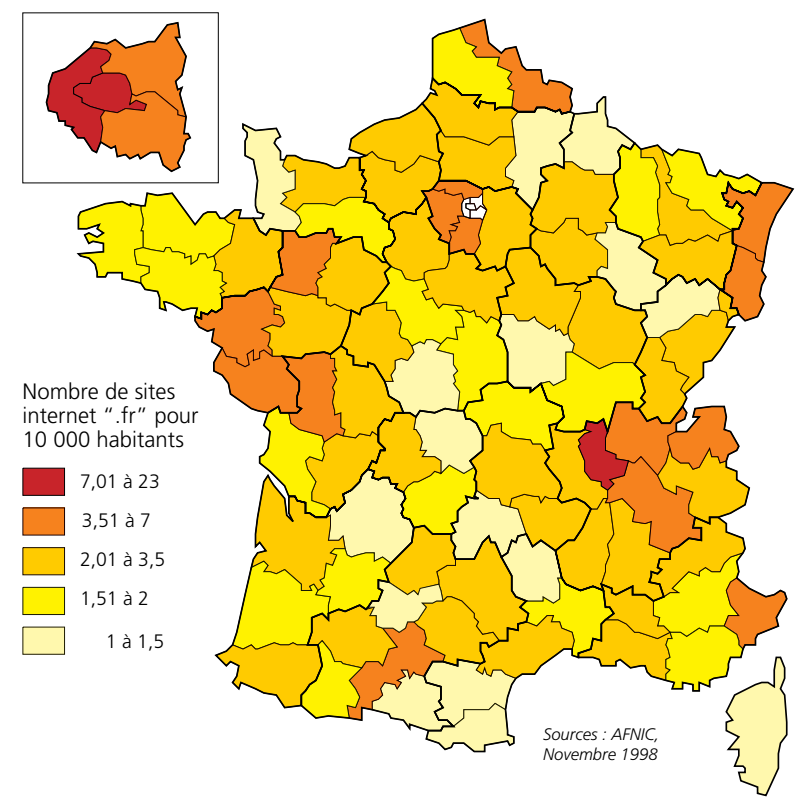

Figure 4.- Nombre de sites Internet "fr" pour 10000 babitants en novembre 1998. 
nombre de sites présents dans ces agglomérations. Le nuage de points représentant la position de chaque ville en fonction de sa taille et du nombre de sites présente une forme allongée (figure 5) montrant une relation apparemment proportionnelle.

Une première régression linéaire est effectuée sur 361 ZAU. Afin de rendre plus significative notre étude, nous avons extrait la ZAU de Paris qui écrase la relation (comme souvent). Une seconde régression montre une relation forte entre la taille et le nombre de sites présents (avec un $\mathrm{R}^{2}=0.78$ ). Pour étudier la relation de façon plus précise, nous avons segmenté le nuage de points en plusieurs souspopulations en retenant des seuils de taille fréquemment utilisés.

Pour les ZAU inférieures à 25000 habitants, la corrélation est nulle, ce qui implique qu'il n'y a pas de liaison entre la taille et le nombre de sites. Pour les trois sous-populations suivantes (de 25000 à 50000 , de 50000 à 100000 , et de 100000 à 200 000) la liaison s'améliore au fur et à mesure, mais dans une proportion moindre. Au-delà de 200000 habitants, la corrélation est significative, confirmant la relation (taille-sites). Nous avons éliminé les ZAU de plus de 400000 habitants soit 14 ZAU car elles n'ont pas tout à fait le même comportement et nous nous sommes penchés plus particulièrement sur le comportement des villes de taille inférieure.

La distribution spatiale des résidus de la régression présente une structure assez significative. En effet, on retrouve des régions avec de forts résidus négatifs. Il s'agit du département du Nord et plus particulièrement des ZAU du Bassin Houiller, de la Lorraine dans sa très grande majorité ainsi que des Ardennes qui sont des zones au tissu industriel en pleine reconversion. Le centre de la France se distingue par des valeurs négatives plus faibles ainsi que tout le Sud-Ouest et le delta du Rhône. Enfin, la Seine-Maritime ainsi que la majorité des ZAU bretonnes présentent aussi des résidus négatifs (figure 6). À l'inverse, les régions RhôneAlpes, l'Alsace et une écharpe qui va de l'Eure à la Vendée montrent des résidus positifs. Ces régions sont caractérisées par un tissu industriel de petites et moyennes entreprises ce qui pourrait être un des facteurs explicatifs du nombre plus important de sites web.

Une étude plus poussée sera entreprise pour tenter de décrire les sites (d'entreprises, de collectivités, à but de promotion touristique ou simplement personnel) afin de mieux cerner le phénomène et d'introduire des facteurs explicatifs plus pertinents. En outre, cette analyse n'est pas complète puisqu'il faudrait aussi analyser la localisation des sites ".com", ".org", et ".net". Toutefois, les sites ".com" sont certainement eux aussi implantés en milieu urbain puisqu'ils dépendent directement de l'activité économique. Il est donc vraisemblable que le phénomène est encore plus lié au réseau urbain que ne le laissent penser les quelques mesures réalisées ci-dessus.

Comme pour les autres innovations technologiques (Saint-Julien, 1985), la diffusion d'Internet s'opère au sein du réseau urbain français "du haut vers le bas" dans la hiérarchie des agglomérations, bien qu'il y ait moins de contraintes financières et techniques pour s'équiper, car la création et la maintenance d'un site sont peu onéreuses. 


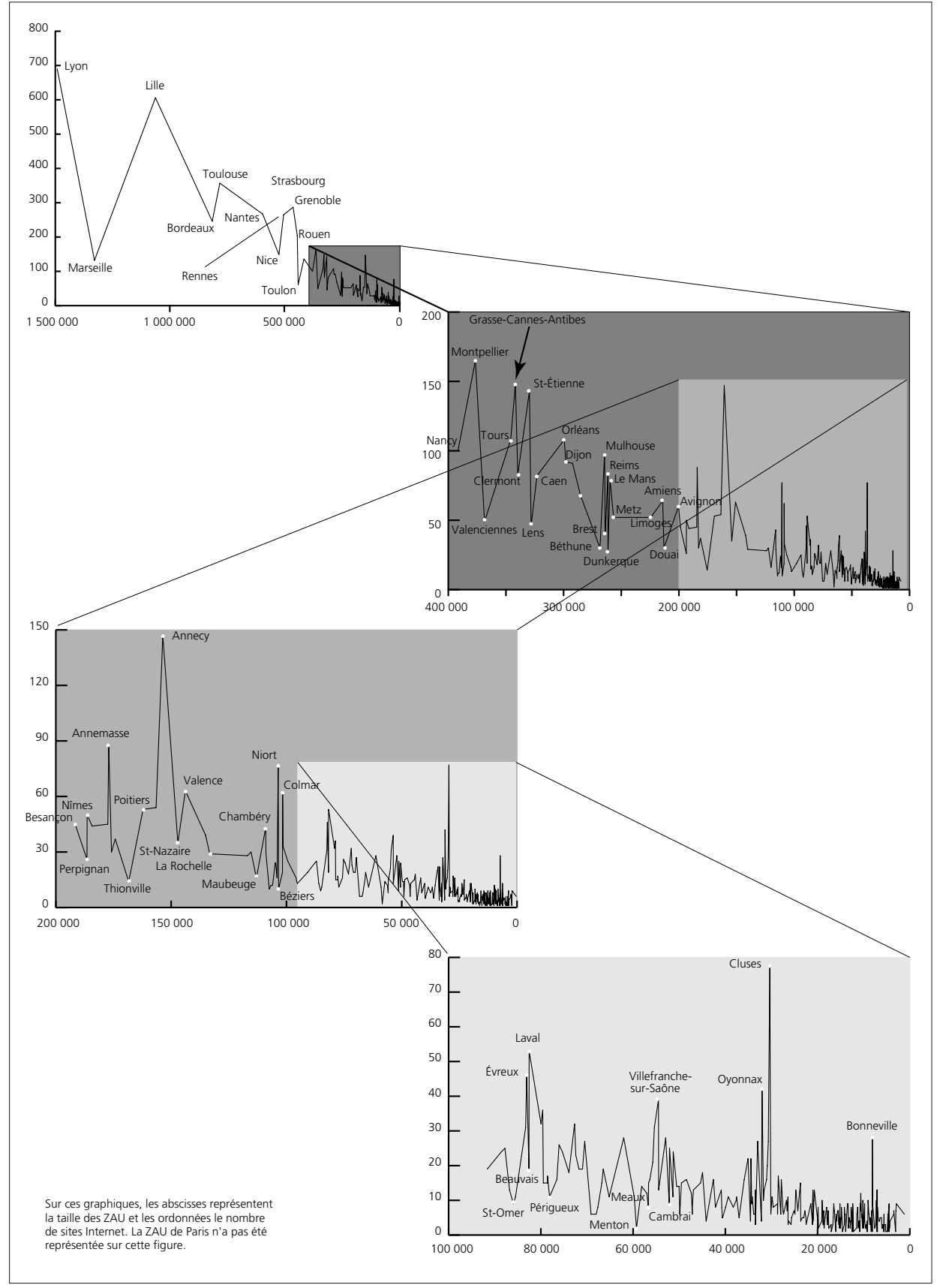

Figure 5.- Taille des ZAU et nombre de sites Internet (hors Paris). 


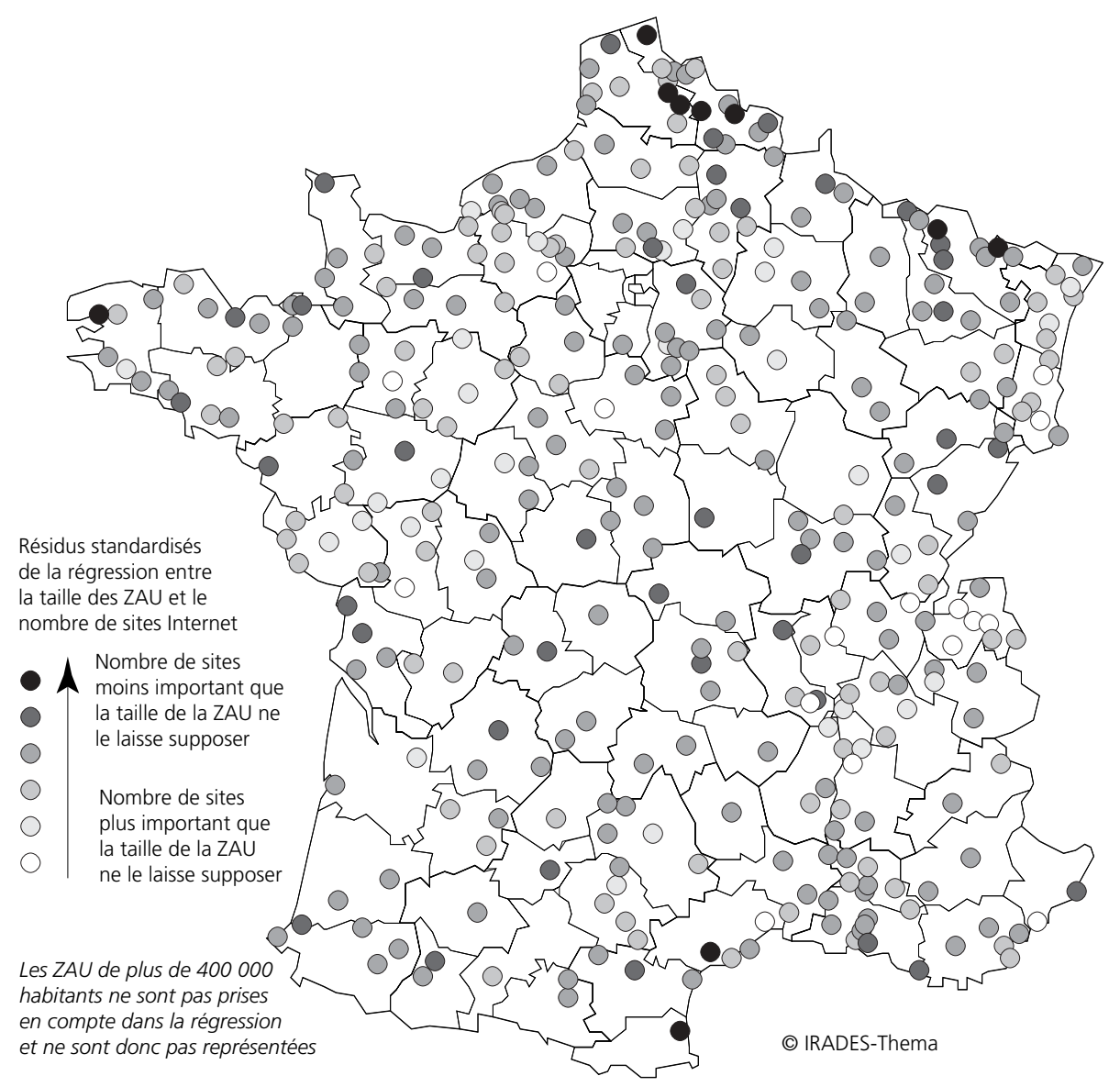

Figure 6.-Localisation des résidus de la régression entre taille et nombre de sites.

Après avoir localisé les fournisseurs et les sites, il convient maintenant de s'attarder plus particulièrement sur les infrastructures lourdes, c'est-à-dire des équipements extrêmement coûteux et donc rares, capables de supporter des trafics d'information à hauts débits.

\section{Des infrastructures exclusivement urbaines : les téléports et les réseaux métropolitains}

Les téléports et les réseaux métropolitains en fibre optique (ou MAN, metropolitan area network) représentent à l'heure actuelle les deux types d'infrastructures de télécommunication les plus coûteux, mais permettant le développement d'applications à hauts débits (supérieurs à 2 mégabits). La diffusion de la technique 
de l'ADSL 5 et des boucles locales radio bouleverseront peut-être cette situation, mais nous n'avons retenu que ces deux types d'infrastructures, notamment parce qu'ils font l'objet de débats en relation avec l'aménagement du territoire et qu'ils polarisent des tensions entre les principaux acteurs que sont France Telecom et les collectivités territoriales. L'exemple du procès intenté au "Grand Nancy" par France Telecom illustre cet enjeu (La Lettre des Télécommunications, n 31).

\section{Les téléports, première tentative de contournement de l'opérateur France Telecom}

La World Teleport Association (WTA) définit le téléport comme une plateforme de services intégrés de télécommunications qui fournit à ses utilisateurs, locaux ou distants, des accès rapides, et au meilleur coût, aux réseaux de télécommunications, nationaux et internationaux, qu'ils soient satellites, filaires ou hertziens.

En 1987, face à la demande de nombreuses collectivités territoriales, France Telecom propose le principe de zones de télécommunications avancées (ZTA), c'est-à-dire un aménagement spécifique des tarifs pour les entreprises, réservé à des zones géographiquement circonscrites, mais ces tarifs sont peu attractifs. En 1994, le principe des ZTA est abandonné compte tenu de l'apparition de nouveaux opérateurs. En effet, la libéralisation du secteur lancée en 1990 par l'Union européenne a engendré une diversification des opérateurs avec la venue de grossistes et semi-grossistes, d'opérateurs alternatifs jouant le rôle de concentrateurs, de câblo-opérateurs pouvant offrir des services de téléphonie, ainsi que des opérateurs locaux de télécommunications qui possèdent leur propre infrastructure (Huet, 1995). En 1999, une douzaine de téléports (cf. annexe 1) fonctionnent ou sont des projets avancés.

Un certain nombre de téléports ont adopté un schéma de développement de type métropolitain, c'est-à-dire qu'ils se sont positionnés à l'interface des réseaux internationaux et d'un réseau métropolitain en fibre optique desservant une agglomération. Ils possèdent alors la maîtrise de l'infrastructure régionale de télécommunications et deviennent à ce titre l'interlocuteur privilégié des entreprises de l'agglomération. Avec la nouvelle loi de Réglementation des télécommunications (juillet 1996), l'État accorde des licences expérimentales pour des téléports. Ceux de Marseille-Provence et de Roubaix (Eurotéléport) n'avaient pas attendu l'introduction de cette loi pour démarrer leur activité.

\section{Les réseaux métropolitains (MAN), nouvel outil du contournement?}

Second type d'infrastructures, les réseaux métropolitains à haut débit sont constitués par une boucle en fibre optique reliant des utilisateurs publics ou privés localisés dans une même agglomération. En France, le premier réseau de ce type a été constitué à Besançon, en 1994, par la Municipalité et l'Université de

5. ADSL: Asymetric digital subscriber line ou Réseau de raccordement numérique asymétrique. Il permet de multiplier les débits sur une simple paire de fils de cuivre (raccordement actuel des lignes téléphoniques classiques). France Telecom va lancer une expérimentation en juin 1999 (La Lettre des télécommunications, $\mathrm{n}^{\circ} 34$ ) 
Franche-Comté en vue de relier les bibliothèques publiques et universitaires entre elles et dans un second temps de diminuer les coûts de télécommunications, l'opérateur national ne proposant que des solutions plus onéreuses (Ville de Besançon, 1998). En 1999, de nombreuses institutions publiques se sont interconnectées (rectorat, lycées, collèges, l'état-major militaire, le conseil régional, la Police nationale, etc.).

Près d'une centaine de villes, dont Colmar, Mulhouse ou Valenciennes, ont obtenu des autorisations du ministre chargé des Télécommunications en application de la loi n 90-1170 du 29 décembre 1990 afin de créer leur propre réseau indépendant. Dans d'autres cas, plusieurs villes se sont fédérées pour constituer un réseau comme dans le cas du réseau Cristal en Alsace.

La diffusion des NTIC, plus précisément celle des infrastructures telles que les téléports ou les réseaux métropolitains, n'a pu s'effectuer sans l'intervention de puissants acteurs publics. Des acteurs tels que l'État et les collectivités territoriales ont mis au point des stratégies pour accompagner et développer l'expansion des équipements et des réseaux, mais aussi de services. Depuis 1994, l'État a lancé des appels d'offres visant à susciter des initiatives de la part des secteurs public et privé.

\section{LE RÔLE DES ACTEURS PUBLICS}

\section{Des projets initiés par la puissance publique}

L'État est en effet prêt à encourager les NTIC en créant "un environnement favorable au développement de cette industrie en levant les verrous qui bloquent son développement et pour orienter les aides à la recherche des entreprises vers quelques technologies prioritaires en matière d'information et de communication" (extrait du Programme d'action gouvernemental pour la société de l'information PAGSI, 1998). Les incitations de l'État se traduisent par le lancement d'appel à propositions. L'État apporte alors son soutien aux initiatives en participant directement à leur mise en œuvre, ou en octroyant un label aux projets répondant à certains critères, ou encore en finançant une partie de ceux-ci. L'année 1994 marque une véritable prise de conscience de l'État qui lance alors les premiers appels à propositions intitulés "les autoroutes de l'information".

\section{Les appels à propositions depuis 1994}

Les tableaux (cf. annexes 2 et 3) présentent l'ensemble des appels à projets concernant la société de l'information lancés par des ministères, des organismes publics ou des collectivités territoriales. Ces listes d'appels à proposition montrent bien l'engagement de l'État dans l'expérimentation des NTIC. Pour réaliser ces projets, la loi n 96-299 instaure un régime de "licence expérimentale". "Des projets d'expérimentation, parmi les plus innovants, et portant sur des plates-formes régionales d'infrastructures expérimentales, vont pouvoir être réalisés en dérogation à certaines dispositions du code des postes et télécommunications (...) comme la 
réalisation et la gestion de plates-formes offrant un large éventail de services avancés de télécommunications sur une zone donnée, et l'utilisation des réseaux câblés de télédistribution pour la fourniture du service téléphonique au public" (D'Attilio, 1998a).

Les autorisations visent l'établissement et l'exploitation d'infrastructures afin de fournir au public, sur des sites géographiquement limités et pour la desserte d'un nombre maximum de 20000 utilisateurs, tous services de télécommunications, y compris le service téléphonique entre points fixes et la fourniture, sur des réseaux établis ou exploités en application de la loi n 86-1067, de tous services de télécommunications, y compris du service téléphonique entre points fixes (D’Attilio, 1998a).

Sur 635 dossiers déposés, 244 projets seront labellisés d'intérêt public en 1995 et 1996 et bénéficieront donc d'une aide de l'État. L'éventail des projets est large puisqu'il recouvre aussi bien des projets d'intérêt général (enseignement, santé, culture, recherche, administration) que des projets à but lucratif (commerce électronique, presse, transport, tourisme, téléservices) ou encore des projets fédérateurs (30 grandes plates-formes régionales d'infrastructures et 3 grands projets nationaux). Une analyse par thèmes dominants traduit la richesse et les nombreux domaines d'intervention des NTIC (tableau 1).

La localisation des initiatives fournit une image assez conforme à ce que nous avons vu pour la diffusion du web. Les régions les plus urbanisées concentrent le plus de projets, mais exceptionnellement la région Provence-Alpes-Côte d'Azur devance l'Île-de-France et Rhône-Alpes. Midi-Pyrénées (avec Toulouse), l'Aquitaine (Bordeaux) et la Lorraine présentent un nombre relativement important de projets (figure 7). Une meilleure prise de conscience de certains acteurs régionaux s'est traduite par le fait que certaines régions ont investi davantage dans ce domaine. La Lorraine, par exemple, a utilisé ces nouvelles technologies afin d'améliorer son image caractérisée par le "déclin industriel".

Les projets d'intérêt national (84) sont les plus nombreux. Ils peuvent s'appliquer à tout le territoire et sont la plupart du temps lancés par les grands services de l'État. La francophonie n'a pas été oubliée dans ces initiatives puisque la plupart des projets de dimension internationale lui sont consacrés (Eveno, 1998).

\section{Le Programme d'action gouvernemental pour la société de l'information (1998-1999)}

Après l'expérimentation de l'appel à proposition lancé sur les autoroutes de l'information, l'État renforce encore son rôle. Les

Tableau 1.-Classement des projets en fonction du thème dominant.

\begin{tabular}{|lr|}
\hline Thème dominant & $\begin{array}{r}\text { Nombre } \\
\text { de projets }\end{array}$ \\
\hline Plate-forme & 70 \\
Télé-enseignement/Éducation & 39 \\
Télé services & 36 \\
Culture & 15 \\
Place de marché & 15 \\
Audiovisuel & 14 \\
Recherche & 14 \\
Presse & 13 \\
Administration & 10 \\
Transport & 8 \\
Santé & 6 \\
Tourisme & 4 \\
\multicolumn{2}{|c|}{ Sources: Autoroutes et services de l'information, } \\
liste des 170 premiers projets labellisés et \\
Autoroutes et services de l'information, liste des \\
74 projets labellisés supplémentaires. \\
\hline
\end{tabular}


principales mesures prônées sont présentées dans un texte de référence: le PAGSI. Six axes prioritaires d'action ont été retenus: l'école, la culture et ses contenus, la modernisation des services publics, les entreprises et le commerce électronique, l'innovation et la régulation.

Pour parvenir aux objectifs qu'il s'est fixés, l'État investit 5,76 milliards de francs sur deux ans (2,12 milliards en 1998 et 3,6 milliards en 1999). D'après le Comité interministériel pour la société de l'information (CISI), sur 218 objectifs désignés début 1998, 70 \% ont été atteints et d'autres sont majoritairement en cours de réalisation.

L'État est intervenu directement au travers de ses services (les rectorats pour l'enseignement, les CHU pour la médecine, etc.). Cependant, les partenaires privés n'en sont pas exclus, comme le montre le rôle majeur de France Telecom, et plus généralement d'entreprises dans le domaine des télécommunications ou fortement utilisatrices de ces technologies.

Pour certains projets, des structures mêlant intérêts publics et privés se sont constituées afin de les réaliser: l'exemple des téléports est tout à fait révélateur de cette situation. Ainsi des projets de téléports sont soutenus majoritairement par des acteurs publics de l'aménagement territorial (conseils régionaux ou généraux, villes ou communautés urbaines) qui ont une vision de développement économique local. Des structures représentatives du monde économique, chambres de commerce et d'industrie (CCI), participent fréquemment aux projets.

Enfin, les collectivités territoriales se sont investies seules dans certains projets, et ont fortement contribué à la diffusion des NTIC et aux moyens de mise en ouvre d'infrastructures.

\section{Un investissement fort des collectivités territoriales}

L'investissement des collectivités territoriales a débuté dans une perspective de développement local et se renforce avec leur rôle de gestionnaire des infrastructures de télécommunications sur leur territoire qui leur est attribué par le décret nº 97-683 du 30 mai 1997.

\section{La gestion des réseaux de télécommunications: une nouvelle compétence}

En effet, à partir du 1er janvier 1998, les collectivités sont tenues de délivrer des permissions de voirie à tous les opérateurs qui souhaitent faire passer leurs câbles sur le domaine public, en faisant respecter les conditions prévues par la loi:

- les opérateurs y compris France Telecom doivent faire une demande de permission de voirie pour tous travaux aériens ou souterrains. Le maire dispose de deux mois pour répondre, passé ce délai, la permission est réputée accordée. Elle ne peut être refusée que pour des motifs listés dans l'article du décret d'application;

- le maire peut demander à plusieurs opérateurs voulant installer des infrastructures sur le domaine public routier de se rapprocher afin de partager dans des conditions à convenir des infrastructures existantes, évitant ainsi des travaux supplémentaires; 


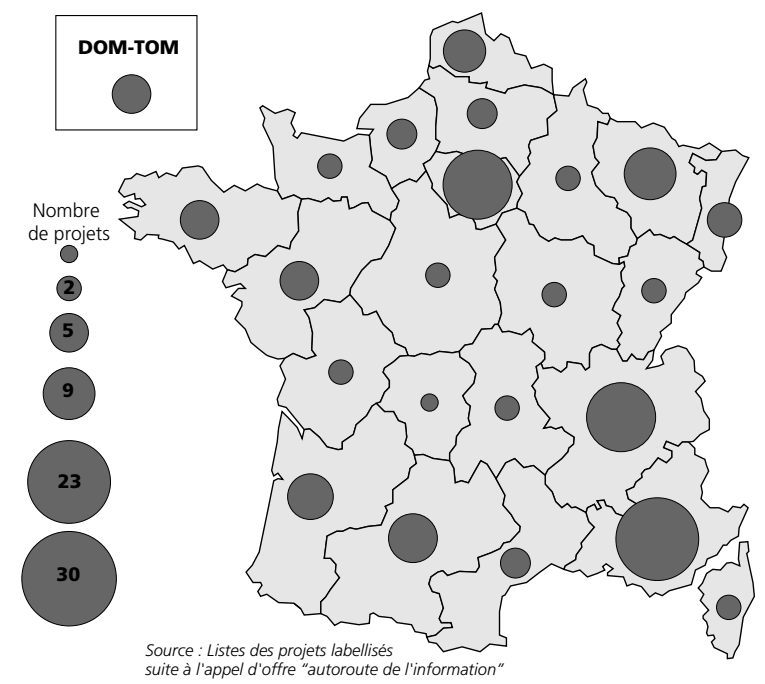

Figure 7.- Nombre de projets labellisés par région, suite à l'appel d'offres "Autoroutes de l'information" de 1994.

- le maire peut aussi demander à l'opérateur propriétaire d'infrastructures de prendre en charge leur déplacement dans le cadre des travaux si l'intérêt du domaine occupé est en cause.

- l'occupation du domaine public donne lieu à redevance à partir du

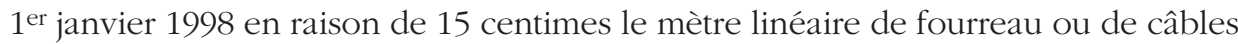
souterrains et par ensemble de câbles entre deux supports en aérien;

- il doit y avoir égalité de traitement entre toutes les demandes.

\section{Une prise de conscience des collectivités}

Toutefois, les collectivités territoriales se sont investies dans les NTIC bien avant que la loi ne le leur impose. Bien que les télécommunications ne figurent pas explicitement dans les compétences communales, départementales et régionales, l'importance de l'utilisation des nouvelles technologies dans l'ensemble des champs d'action couverts par les collectivités locales justifie l'intérêt qu'elles y portent.

Comme le souligne le rapport D'Attilio (1998b), pour les collectivités locales les enjeux sont de nature "politique" au sens large du terme. Les élus ont compris qu'ils disposaient au travers des NTIC d'une technologie transversale, adaptable à leur situation, à leur contexte et à leurs priorités. Que ce soient les maires de grandes agglomérations ou bien le maire d'un petit village chacun d'eux a adapté la construction de son projet à ses moyens et aux objectifs politiques prioritaires qu'il s'est fixés. Les NTIC permettent de donner une cohérence aux politiques que les élus souhaitent mener au service des citoyens qui deviennent acteurs des projets, mais aussi au service du développement local et de l'aménagement du territoire avec comme enjeu majeur l'emploi. 
L'évolution induite par la libéralisation du marché des télécommunications, la perception des enjeux des NTIC dans l'aménagement du territoire par les acteurs publics, notamment, expliquent en grande partie les débats actuels sur le rôle de ces derniers. En d'autres termes, la concurrence trouve toute sa pertinence en ce qui concerne l'aménagement du territoire en France.

\section{PERCÉE CONCURRENTIELLE ET AMÉNAGEMENT DU TERRITOIRE}

Dans un environnement marqué par le foisonnement des nouvelles technologies de l'information et de la communication, le rôle joué par la concurrence est de plus en plus déterminant, notamment vis-à-vis des infrastructures coûteuses que sont les réseaux en fibre optique. En l'absence d'une véritable concurrence sur ce marché des équipements locaux, les collectivités locales se sont impliquées dans la gestion des projets d'infrastructures des télécommunications, en plus de leurs fonctions de développement économique, social et culturel de leur territoire. Le statut juridique des projets d'infrastructures de télécommunications portés par les collectivités territoriales traduit, en partie, la nouvelle fonction de gestionnaire. Nous en verrons les différentes formes d'organisation possibles.

Ces profondes mutations introduites par la libéralisation du secteur des télécommunications en France ont eu de grandes répercussions, parfois imprévues, sur l'aménagement du territoire, déclenchant un débat vigoureux.

\section{L'impact de l'ouverture à la concurrence sur l'aménagement du territoire}

Tout a commencé avec la loi du $1^{\text {er }}$ juillet 1996 sur la réglementation des télécommunications. En effet, cette loi n'autorise pas, ni n'interdit, aux collectivités locales de devenir opérateurs. La seule certitude c'est que les collectivités peuvent établir un réseau de télécommunications indépendant (réservé à un usage privé ou indépendant) pour un groupe fermé d'utilisateurs (GFU). Certaines collectivités locales tentent ainsi d'aller plus loin, puisque, en tant que gestionnaire d'infrastructures de télécommunications de leur territoire, elles peuvent désormais être fournisseurs de réseaux aux opérateurs, ce qui constitue une source de revenus supplémentaire et leur permet de mieux maitriser leur développement local.

Le flou juridique laissé par la loi de juillet 1996 nourrit actuellement une polémique entre France Telecom et des collectivités territoriales qui ont manifesté leur mécontentement dans un mémorandum adressé au Premier ministre (Mémorandum, 1999). Les collectivités territoriales se plaignent du monopole de l'opérateur au niveau de la boucle locale, ce qui les empêche de mettre en place un réseau et de nouveaux services. L'opérateur, confronté depuis peu à l'intervention de Cegetel sur la boucle locale, se plaint d'une possible concurrence déloyale de la part des collectivités territoriales qui risquerait à terme de favoriser certains opérateurs de télécommunications, ou certaines entreprises (en leur offrant des tarifs attractifs). 
Dans le Mémorandum, les collectivités territoriales rejettent cette accusation et "affirment clairement qu'elles n'entendent pas jouer un rôle d'opérateur télécom tel qu'il est défini par la loi de juillet 1996 car elles considèrent que c'est un métier devant être exercé par des entreprises professionnelles. Elles veulent jouer uniquement leur rôle d'aménageur en développant sur leurs territoires des infrastructures passives de télécommunications appelées plus communément fibre noire. Le service offert par les collectivités territoriales, dans ce contexte, relève d'un service de gestion d'infrastructures [...] Ces réseaux sont destinés à créer les conditions d'accueil des opérateurs télécom nouveaux et traditionnels, sans discrimination, et dans le respect des règles de la concurrence. Ils font l'objet d'un mandat de gestion auprès de professionnels qui ne détiennent pas de licences d'opérateurs".

\section{Les formes juridiques adoptées pour la gestion d'un réseau :}

Pour développer ces infrastructures, les collectivités territoriales ont mis en place des organismes capables de gérer leurs propres réseaux échappant au contrôle de l'opérateur historique France Telecom. Le choix du statut juridique a été multiple, ce qui traduit, d'une part, toute la difficulté que ces promoteurs ont rencontrée pour arrêter leur choix et, d'autre part, leur degré d'investissement.

En tout cas, les projets d'infrastructures de télécommunications aujourd'hui mis en place accordent une attention tout à fait particulière à la définition du cadre juridique. En effet, s'impliquer dans un projet d'infrastructures de télécommunications est une décision importante pour une collectivité qui relève, dans la plupart des cas, soit d'une carence (absence d'offre commerciale de la part des opérateurs de télécommunications), soit d'une volonté d'agir plus directement encore sur le développement économique, social et culturel de son territoire. Ainsi, l'analyse de l'origine du projet d'infrastructures des télécommunications pour une collectivité territoriale constitue un bon indicateur puisqu'il peut justifier l'adoption du statut juridique à mettre en place.

\section{La gestion publique directe (GPD)}

Il s'agit d'un mode de gestion directe d'un réseau de télécommunications par une collectivité locale. Ce mode de gestion va de pair avec le développement de l'informatique communicante et des réseaux, et fait naître des projets, où les NTIC touchent aux compétences administratives. Par exemple, le "Réseau Lumière" de fibre optique déployé sur la ville de Besançon regroupe cinq collectivités publiques de manière informelle. La structure fonctionne à l'intérieur d'un groupe fermé d'utilisateurs (GFU) qui a été autorisé par l'Autorité de régulation des télécommunications (ART). Ce réseau est financé par les pouvoirs publics et se restreint pour le moment au domaine public. 
La société d'économie mixte locale (SEML)

Ce type de société associe une collectivité publique (État, commune, département, région) à des capitaux privés pour réaliser des opérations d'aménagement et de construction, pour exploiter des services publics à caractère industriel ou commercial, ou pour toute activité d'intérêt général. La société doit nécessairement revêtir la forme d'une société anonyme et les collectivités locales doivent détenir plus de la moitié du capital et plus de la moitié des voix dans les organes délibérants. C'est la loi du 7 juillet 1983 qui a permis la création de sociétés d'économie mixte locales. La ville de Castres a mis en place une SEML pour gérer son réseau de télécommunications.

\section{La société anonyme d'économie mixte locale (SAEML)}

Ce type de société se compose aussi de capitaux privés et de capitaux publics, mais les membres doivent être au moins au nombre de sept. Le pouvoir décisionnaire de chaque membre est proportionnel à son apport. La collectivité doit être majoritaire. Dans le cas du réseau Cristal, le choix de la région porte sur une SAEML, et réunit à son capital la communauté urbaine de Strasbourg, les villes de Strasbourg, de Mulhouse, de Colmar, les départements du Bas-Rhin et du HautRhin, la région Alsace et de nombreuses sociétés privées.

\section{Le groupement d'intérêt économique (GIE)}

Le GIE est une personne morale ayant une pleine capacité juridique. Ce type d'organisation n'est pas seulement réservé aux commerçants. Il doit avoir pour objet le prolongement de l'activité économique de ses membres. Il n'a pas pour but de réaliser des bénéfices, mais il ne lui est pas interdit d'en réaliser et s'il y a en, ses membres se les partagent. La constitution d'un GIE exige aux moins deux membres. La ville de Colmar a mis en place un GIE qui regroupe la régie municipale de Colmar, l'IUT et la CCI. Un consortium associant des entreprises privées complète le dispositif. Le GIE de Colmar a pour vocation de mettre en ouvre tous les moyens propres à faciliter et à développer l'activité économique de ses membres.

L'intervention des collectivités territoriales dans l'établissement de réseaux d'infrastructures traduit la prise de conscience de celles-ci vis-à-vis des capacités d'aménagement qu'apportent ces techniques.

\section{Les enjeux des NTIC pour les collectivités locales dans l'aménagement de leur territoire}

Les NTIC ont fait irruption dans le débat sur l'aménagement du territoire au nom de l'égalité d'accès des entreprises et des citoyens à ces techniques sur le territoire. Comme nous avons pu le voir au travers de la répartition des utilisateurs comme des infrastructures, ce principe est loin d'être appliqué, les zones de faible densité n'ayant pas accès à des infrastructures de qualité. Les collectivités territoriales ont donc développé un argumentaire s'appuyant sur cette carence pour tenter d'avoir une plus grande liberté de manœuvre dans la gestion de ces NTIC. À partir 
Tableau 2.-Quelques modes de gestion de réseaux de Télécommunications.

\begin{tabular}{|c|c|c|c|}
\hline Nom du projet & Villes & Structure juridique & Partenaires publics \\
\hline $\begin{array}{l}\text { Réseau Lumière } \\
\text { (vocation non commerciale) }\end{array}$ & Besançon & $\begin{array}{l}\text { GPD } \\
\text { (direction de l'informatique) }\end{array}$ & 5 \\
\hline $\begin{array}{l}\text { Oasice } \\
\text { (vocation non commerciale) }\end{array}$ & Colmar & GIE & 3 \\
\hline $\begin{array}{l}\text { Anneau numérique } \\
\text { (vocation commerciale) }\end{array}$ & Castres & SEML & $4+$ privés \\
\hline $\begin{array}{l}\text { Cristal } \\
\text { (vocation commerciale) }\end{array}$ & Région Alsace & SAEML & $\begin{array}{l}\text { Collectivités régionales + } \\
\text { Privés }\end{array}$ \\
\hline
\end{tabular}

du Mémorandum adressé au Premier ministre ainsi que d'une analyse conduite par D'Attilio, nous relevons l'argumentaire utilisé par les collectivités territoriales.

\section{Les NTIC, facteurs de localisation et actrices de l'aménagement?}

Le premier argument avancé par les collectivités a trait à la relation entre NTIC et accessibilité. Les collectivités territoriales ont souligné le rôle de plus en plus crucial d'une bonne desserte de leur environnement par des infrastructures de télécommunications performantes pour attirer les entreprises et offrir de meilleurs services à leurs citoyens.

Le critère "télécommunications" semble prendre de l'importance auprès des entreprises même s'il n'est jamais primordial. Il est maintenant souvent retenu dans les facteurs de localisation au même titre que la diversité des transports et la qualité de la main-d'œuvre locale. Or les collectivités soulignent le retard d'une grande partie du territoire français face à d'autres pays européens, mais surtout vis-à-vis des zones de fortes densités de population en France.

La desserte télécom du public résidentiel n'est pas non plus étrangère aux préoccupations des collectivités locales. Là encore, les élus avancent le risque de voir se créer de nouveaux facteurs d'exclusion, géographiques ou économiques, faisant des zones à forte densité, ou à forte potentialité de trafic, les seules bénéficiaires des effets positifs de la concurrence. De la même façon, ils ne peuvent admettre l'émergence de nouvelles ruptures sociales consécutives à des inégalités d'accès aux technologies de la communication, donc aux savoirs, revenant ainsi au mythe de l'égalité entre les citoyens.

Les NTIC sont aussi perçues comme un véritable outil d'aménagement du territoire. Les responsables politiques locaux considèrent que les NTIC constituent un formidable facteur de développement local, permettant une structuration du territoire plus équitable et permettant de se rapprocher des citoyens (démocratie locale) à des coûts relativement modestes. Les collectivités locales pensent pouvoir jouer un rôle déterminant dans l'accélération de la mise en place de la société de l'information. Elles sont un catalyseur important de l'accès "grand public" aux 
réseaux via Internet mais également au développement des usages et de leur logique, au service du développement de la cohésion sociale par la modernisation des services publics (éducation, santé, démocratie, culture...).

Enfin, les objectifs de maîtrise des coûts de fonctionnement (qui ont fortement augmenté pour les communications locales) et d'investissements télécoms ont plaidé pour la participation des collectivités au déploiement de réseaux de télécommunications en "fibre noire" (fibre optique non activée), surtout depuis que la loi les a désignées seules gestionnaires de leur sous-sol.

\section{Le facteur déterminant: l'absence de concurrence au niveau de la boucle locale?}

Les collectivités justifient leur intervention dans les infrastructures de télécommunications par la carence de la concurrence au niveau de la boucle locale. En effet, les opérateurs de télécommunications privilégient un retour d'investissement rapide des infrastructures à haut débit qu'ils installent, ce qui suppose un trafic important engendré par des entreprises ou par un nombre d'abonnés considérable (zones de fortes densités).

Or comme le souligne le Mémorandum: "L'absence d'offre de l'opérateur historique à des conditions tarifaires avantageuses pour les entreprises, les collectivités territoriales et les services publics sur ce terrain de la boucle locale crée une situation qui conduit au développement de quelques pôles d'excellence sur le territoire face à de véritables friches de télécommunications".

Même en région parisienne, qui pourtant offre un potentiel d'entreprises (courtisées par de grands réseaux privés comme celui de MCI-Worldcom) et de population conséquent, des communes "de la périphérie" ont monté un projet d'infrastructures en fibre noire (projet du syndicat intercommunal de la périphérie de Paris pour l'électricité et les réseaux de communications - SIPPEREC), pour permettre une meilleure concurrence et donc des tarifs plus avantageux. Le déploiement d'une infrastructure en "fibre noire" par les collectivités territoriales est donc un des supports nécessaires à l'existence d'une véritable concurrence entre opérateurs sur leur territoire, sans laquelle les déséquilibres au sein de leur environnement ne pourront que s'aggraver.

Les zones de faible densité économique et de population ont bien compris que seule une initiative de leurs collectivités territoriales pourra leur fournir les équipements nécessaires à leur développement. C'est pourquoi certains élus manifestent le souhait de se regrouper pour financer des infrastructures de type "fibre noire" et prendre à leur charge le surcoût lié à la localisation sur leur territoire, c'est-à-dire lié à l'éloignement des réseaux de communications à haut débit des opérateurs privés de télécommunications pour servir des entreprises qui souhaiteraient s'installer chez eux. 
Une convergence entre les nouvelles lois liées à l'aménagement du territoire et la gestion collective des réseaux?

Un projet de loi ( $n^{\circ} 1155$ de février 1999, relatif à l'organisation urbaine et à la simplification de la coopération intercommunale) vise à améliorer la coopération intercommunale en France qui, jusqu'à présent, a montré des résultats mitigés. Les élus de l'Assemblée nationale ont conscience que le tissu communal français est un héritage lourd à gérer et inadapté à une bonne gestion du territoire. Ils ont constaté un relatif échec des politiques de regroupement volontaristes, déclinées notamment sous forme d'incitations aux fusions de communes.

Ce projet prend appui sur la définition des villes en aires urbaines (ZAU), récemment mise au point par l'INSEE. Il vise à renforcer la cohésion territoriale ainsi qu'à simplifier le droit existant:

- il s'agit de créer plus de solidarité en milieu rural et de favoriser l'émergence des agglomérations. Le regroupement impliquerait plus de solidarité par le partage de la ressource fiscale et des transferts financiers aux communes les plus défavorisées;

- l'objectif est de rationaliser les structures intercommunales en supprimant les communautés de villes, les districts, et en créant des communautés d'agglomération pour les petites et moyennes villes et des communautés urbaines pour les très grandes villes dont les compétences sont mieux définies.

Cette échelle de gestion territoriale, préconisée dans la loi, correspond en fait assez bien à celle d'un réseau de télécommunications. En effet, l'échelle des enjeux en matière de réseau de télécommunications impose de raisonner sur des plans intercommunaux ou départementaux. Ceux-ci seuls assurent la cohérence des interventions et des investissements sur un territoire homogène. Cet argument est utilisé par les collectivités territoriales pour justifier leur intervention.

\section{CONCLUSION}

L'analyse de la diffusion des nouvelles technologies de l'information et de la communication (NTIC) en France s'est faite au travers de la spatialisation des fournisseurs d'accès à Internet (FAI) et des sites web. Elle confirme le lien étroit qu'entretiennent les NTIC avec le milieu urbain. En effet, presque tous les FAI sont installés en ville et près des trois quarts des sites web sont présents dans les ZAU. Toutefois, la localisation des sites n'est pas neutre puisque l'on repère des villes fortement "sous-équipées" notamment dans le Nord-Est français, ainsi que toute la partie centrale et le Sud-Ouest. En revanche, l'Alsace, Rhône-Alpes et une partie de l'Ouest français présentent un taux d'équipement supérieur. À l'heure actuelle, les facteurs expliquant ce phénomène ne sont pas totalement maîtrisés. La nature des données disponibles est assez hétérogène et d'une fiabilité limitée.

Cette utilisation des NTIC nous a amenés à étudier la répartition des infrastructures "lourdes" qui sont localisées presque exclusivement en milieu urbain. 
Cette concentration s'explique par le contexte concurrentiel encore embryonnaire sur la boucle locale et par le rôle des acteurs publics.

L'État n'intervient jamais directement dans l'installation des réseaux mais sa politique volontariste d'appels d'offres a permis aux collectivités territoriales, notamment, de prendre conscience des enjeux de ces nouvelles technologies. Celles-ci ont compris tout l'intérêt de ces outils pour l'aménagement du territoire et mènent actuellement une lutte pour obtenir la possibilité d'installer et de gérer des réseaux de télécommunications présentant des débits suffisamment élevés. Leur argumentation met en avant la notion d'égalité des citoyens et des entreprises pour l'accessibilité aux NTIC, tout en cherchant aussi à minimiser le coût des télécommunications essentiellement basé sur le tarif local. De plus, la législation leur attribue maintenant un rôle de gestionnaire.

Cette accessibilité est d'autant plus délicate que le territoire est de faible densité et qu'il ne regroupe pas d'entreprises. En effet, les opérateurs de télécommunications concurrents de l'opérateur national n'ont, à l'heure actuelle, pas les moyens financiers de développer des infrastructures sur tout le territoire. Ils se contentent donc de s'implanter dans la partie la plus intéressante, permettant un retour sur investissement rapide. Les collectivités territoriales sont les seules à pouvoir investir et donc réduire les inégalités dans les zones délaissées par les opérateurs. Reste toutefois le problème de la forme juridique à adopter pour réaliser leur pari.

\section{BIBLIOGRAPHIE}

Аммі C., 1997, La Concurrence dans les télécoms, stratégies et perspectives, Paris, Hermès, 247 p. Bratzel S., Dienel L., 1999, National urban systems and ICT in Europe: some comparative remarks, rapport d'étape pour le contrat européen Telecityvsion, 39 p.

D'Atтilio H., 1998a, Le Développement des Nouvelles technologies d'information et de communication dans les collectivités locales: de l'expérimentation à la généralisation, rapport au Premier ministre, $80 \mathrm{p}$.

D’Aтtпlo H., 1998b, Assurer l'égalité des territoires dans l'accès aux Technologies de l'Information et de la Communication pour les zones fragiles, rapport au Premier ministre, $85 \mathrm{p}$.

Dumont B., 1999, "L'ASDL risque de renforcer le monopole de la boucle locale", La Lettre des télécommunications, $\mathrm{n}^{\circ} 34$.

Eveno E., 1998, "Acteurs et territoires de la société d'information: géographie d'un grand chantier français en construction", in Autoroutes de l'information et dynamiques territoriales, dir. A. Lefebvre et G. Tremblay, PUM-Presse universitaire du Québec, p. 285-311.

Gillon P., Caro P., N'Diaye O., Frankhauser P., 1999, Structural analysis of the urban system in France, rapport d'étape pour le contrat européen Telecityvsion, 39 p.

Grasland L.,1997, "Contre l'isolement spatial et pour la valorisation du territoire: l'exemple du web", Montagnes méditerranéennes, n 6, p. 59-67.

GrasLand L., 1998, "Internet et le territoire: un divorce annoncé ou une communauté d'intérêt? L'exemple de la diffusion du web en France", Netcom, vol. 12, n 1,2,3, p. 137-149 
Huet A., Zeitoun J., 1995, Les Téléports: nouvelles places de marché sur les inforoutes, Paris, L'Harmattan, $332 \mathrm{p}$.

LAUBier C., 1999, "Les collectivités territoriales vont pouvoir contrer le monopole de la boucle locale", La Lettre des télécommunications, n 31.

Martin-Lalande P.,1997, L'Internet: un vrai défi pour la France, Rapport au Premier ministre, $90 \mathrm{p}$.

MÉmorandum, 1999, Les collectivités territoriales entendent assumer leurs responsabilités en matière d'aménagement du territoire et d'infrastructures de télécommunications, texte adressé par la Communauté urbaine du Grand Nancy, le Conseil général du Tarn, le District du Grand Toulouse et le Syndicat intercommunal de la périphérie de Paris pour l'électricité et les réseaux de communication (SIPPEREC).

SaINT-Julien Th., 1985, La Diffusion spatiale des innovations, Montpellier, GIP RECLUS, 38 p. Ville de Besançon, 1998, Le Réseau Lumière: premier réseau français métropolitain de télécommunication haut débit, Document de travail, 100 p.

\section{TEXTES ACCESSIBLES SUR LE SERVEUR INTERNET WWW.INTERNET.GOUV.FR (RUBRIQUE TEXTES DE RÉFÉRENCE OU RAPPORTS)}

CISI, Rapport rédigé par le SJTIC, 1999, Mise en œuvre du programme d'action gouvernemental pour la société de l'information, état d'avancement après un an (janvier 1998janvier 1999), $113 \mathrm{p}$.

1998, Appel d'offres relatif aux Autoroutes de l'information 1994-1998.

1998, Autoroutes et services de l'information, liste des 170 premiers projets labellisés.

1998, Autoroutes et services de l'information, liste des 74 projets labellisés supplémentaires.

\section{SITES INTERNET}

www.nic.fr (AFNIC)

www.internet.gouv.fr (Ministères)

www.art-telecom.fr (ART)

http://europa.eu.int/pol/infso/index_fr.htm (Union Européenne)

www.telecomville.org (Observatoire des télécoms dans la ville)

www.cybergeography.org 
Annexe 1.-Descriptif des principaux éléments des projets de téléports en France.

\begin{tabular}{|c|c|c|}
\hline Les téléports en activité & Les partenaires du projet & Type d'opération \\
\hline Eurotéléport de Roubaix & $\begin{array}{l}\text { Partenaires publics } \\
\text { Ville de Roubaix (28,5\%) } \\
\text { Communauté urbaine Lille-Roubaix-Tourcoing (16,5\%) } \\
\text { Conseil général du Nord, ville de Lille, le SIAR }(5,5 \%) \\
\text { Conseil Régional } \\
\text { Partenaires privés } \\
\text { CCI Lille-Roubaix-Tourcoing (5\%) } \\
\text { SARI (promoteur immobilier de la ville) (5\%) } \\
\text { Compagnie Générale des Eaux (5\%) } \\
\text { Caisse d'Épargne (5\%) } \\
\text { Thomson (5\%) }\end{array}$ & $\begin{array}{l}\text { Réhabilitation d'un site } \\
\text { industriel }\end{array}$ \\
\hline Téléport de Marseille-Provence & $\begin{array}{l}\text { Partenaires publics } \\
\text { Ville de Marseille } \\
\text { Conseil général } \\
\text { Conseil régional } \\
\text { Préfecture de Région } \\
\text { Partenaires privés } \\
\text { CCI Marseille Provence } \\
\text { Actionnaires privés (arrivent dans la phase de démarrage) }\end{array}$ & $\begin{array}{l}\text { Intégration dans un projet } \\
\text { d'aménagement du } \\
\text { centre-ville de Marseille } \\
\text { (Euroméditerranée) }\end{array}$ \\
\hline $\begin{array}{l}\text { Île-de-France } \\
\text { (Téléport multisites: } \\
\text { La Défense, Marne-la-Vallée, } \\
\text { Roissy, Plateau de Saclay, } \\
\text { Saint-Quentin-en-Yvelines) }\end{array}$ & $\begin{array}{l}\text { Partenaires publics } \\
\text { Conseil régional } \\
\text { Collectivités locales (Association Téléport IF) } \\
\text { Partenaire privé } \\
\text { France Télécom (depuis 1998) }\end{array}$ & \\
\hline Les téléports en projet & Les partenaires du projet & Type d'opération \\
\hline Brest & $\begin{array}{l}\text { Partenaire public } \\
\text { Communauté urbaine de Brest } \\
\text { Partenaire privé } \\
\text { CCI de Brest }\end{array}$ & $\begin{array}{l}\text { Réaménagement de la } \\
\text { façade portuaire et requa- } \\
\text { lification du front de mer } \\
\text { Synergie et mise en } \\
\text { valeur de ressources } \\
\text { locales dans le secteur } \\
\text { des télécommunications }\end{array}$ \\
\hline Bordeaux & $\begin{array}{l}\text { Partenaires publics } \\
\text { Conseil général } \\
\text { Communauté urbaine de Bordeaux } \\
\text { Partenaire privé } \\
\text { CCI de Bordeaux }\end{array}$ & $\begin{array}{l}\text { Tourné prioritairement } \\
\text { vers la communauté } \\
\text { des affaires }\end{array}$ \\
\hline Auvergne & $\begin{array}{l}\text { Partenaires publics } \\
\text { Association Téléport Auvergne (loi 1901) } \\
\text { Comité d'expansion économique (43) } \\
\text { SIVOM Vivarais-Lignon } \\
\text { Conseil général } \\
\text { Conseil régional }\end{array}$ & En zone rurale \\
\hline Orléans & $\begin{array}{l}\text { Partenaires publics } \\
\text { District de l'Est orléanais } \\
\text { Conseil régional } \\
\text { Partenaire privé } \\
\text { CCI d'Orléans }\end{array}$ & $\begin{array}{l}\text { Intégration à un projet } \\
\text { de technopole }\end{array}$ \\
\hline Strasbourg & $\begin{array}{l}\text { Partenaire public } \\
\text { Communauté Urbaine de Strasbourg } \\
\text { Partenaire privé } \\
\text { CCI de Strasbourg }\end{array}$ & $\begin{array}{l}\text { Réaménagement de la } \\
\text { zone portuaire dans le } \\
\text { cadre de l'implantation } \\
\text { d'une ZAC }\end{array}$ \\
\hline Calvisson La Vaunage & En projet & \\
\hline Metz 2000 & En projet & \\
\hline Sophia Antipolis & En projet & \\
\hline Poitiers (Futuroscope) & En projet & \\
\hline
\end{tabular}


Annexe 2.- Appels d'offres à projets concernant la société de l'information.

\begin{tabular}{|c|c|c|c|}
\hline Intitulé de l'appel & Date & Commanditaire & But principal \\
\hline $\begin{array}{l}\text { Appel à propositions relatif } \\
\text { aux expérimentations des } \\
\text { nouveaux services des } \\
\text { autoroutes de l'information }\end{array}$ & $\begin{array}{l}11 / 1994- \\
01 / 1995\end{array}$ & $\begin{array}{l}\text { Ministère de l'Industrie, } \\
\text { des postes et télécommunications } \\
\text { et du commerce extérieur }\end{array}$ & $\begin{array}{l}\text { Promouvoir de nouveaux services, } \\
\text { de nouvelles applications et de } \\
\text { nouvelles offres de contenu }\end{array}$ \\
\hline $\begin{array}{l}\text { Appel à propositions } \\
\text { dénommé TIC (Technologies } \\
\text { de l'Information et de } \\
\text { la Communication) }\end{array}$ & $\begin{array}{l}02 / 1996- \\
04 / 1996\end{array}$ & $\begin{array}{l}\text { Ministère délégué à la Poste, } \\
\text { aux Télécommunications } \\
\text { et à l'Espace, et l'ANVAR }\end{array}$ & $\begin{array}{l}\text { Soutenir le développement } \\
\text { des nouvelles technologies }\end{array}$ \\
\hline $\begin{array}{l}\text { Appel à propositions } \\
\text { "Recherche et Filtrage de } \\
\text { l'information sur les réseaux" }\end{array}$ & $06 / 1997$ & $\begin{array}{l}\text { Ministère de l'Éducation nationale, } \\
\text { de l'Enseignement supérieur } \\
\text { et de la Recherche et ministère } \\
\text { de l'Industrie, de la Poste } \\
\text { et des Télécommunications }\end{array}$ & $\begin{array}{l}\text { Faciliter l'accès à l'information } \\
\text { électronique et de soutenir le } \\
\text { développement } \\
\text { d'outils innovants }\end{array}$ \\
\hline $\begin{array}{l}\text { Appel à projets } \\
\text { "Territoire et nouvelles } \\
\text { pratiques pédagogiques" }\end{array}$ & $\begin{array}{l}04 / 1998- \\
06 / 1998\end{array}$ & $\begin{array}{l}\text { Ministère de l'Aménagement } \\
\text { du territoire et de l'environnement } \\
\text { et Ministère de la Culture } \\
\text { et de la communication }\end{array}$ & $\begin{array}{l}\text { Encourager l'utilisation des NTIC } \\
\text { pour enrichir les pratiques } \\
\text { pédagogiques dans les } \\
\text { établissements scolaires, notamment } \\
\text { dans les zones rurales et } \\
\text { de reconversion industrielle }\end{array}$ \\
\hline $\begin{array}{l}\text { Appel à projets } \\
\text { "Bibliothèques et nouvelles } \\
\text { technologies de l'information } \\
\text { et de la communication" }\end{array}$ & $\begin{array}{l}04 / 1998- \\
06 / 1998\end{array}$ & $\begin{array}{l}\text { Ministère de l'Aménagement } \\
\text { du territoire et de l'environnement } \\
\text { et Ministère de la Culture } \\
\text { et de la communication }\end{array}$ & $\begin{array}{l}\text { Favoriser l'accès du public aux NTIC, } \\
\text { par l'intermédiaire des bibliothèques } \\
\text { de communes ou groupements } \\
\text { de communes de moins de } \\
5000 \text { habitants }\end{array}$ \\
\hline $\begin{array}{l}\text { Appel à projets } \\
\text { "Téléformation des } \\
\text { TTPMI-PME" }\end{array}$ & $\begin{array}{l}04 / 1998- \\
06 / 1998\end{array}$ & $\begin{array}{l}\text { Ministère de l'Aménagement } \\
\text { du territoire et de l'environnement } \\
\text { et Ministère de la Culture } \\
\text { et de la communication }\end{array}$ & $\begin{array}{l}\text { Préparer les très petites entreprises } \\
\text { aux nouvelles télé-pratiques } \\
\text { professionnelles }\end{array}$ \\
\hline $\begin{array}{l}\text { Appel à propositions } \\
\text { "NTIC et innovations dans } \\
\text { les services" }\end{array}$ & $\begin{array}{l}06 / 1998- \\
07 / 1998\end{array}$ & $\begin{array}{l}\text { Secrétariat d'État à l'industrie } \\
\text { et l'ANVAR }\end{array}$ & $\begin{array}{l}\text { Développer les NTIC qui mettent à } \\
\text { profit les technologies de } \\
\text { l'information et de la } \\
\text { communication et les téléactivités }\end{array}$ \\
\hline
\end{tabular}


Annexe 3.- Appel d'offres à projets concernant la société de l'information (suite).

\begin{tabular}{|c|c|c|c|}
\hline Intitulé de l'appel & Date & Commanditaire & But principal \\
\hline $\begin{array}{l}\text { Appel à projets pour le } \\
\text { développement de la société } \\
\text { de l'information en région } \\
\text { Limousin }\end{array}$ & $\begin{array}{l}04 / 1998- \\
07 / 1998\end{array}$ & $\begin{array}{l}\text { Commission européenne } \\
\text { et Conseil Régional, ouvert } \\
\text { aux services de l'État et aux } \\
\text { organismes consulaires }\end{array}$ & $\begin{array}{l}\text { S'adresse aux associations, } \\
\text { communes et groupes de communes } \\
\text { désireuses d'assurer une mission } \\
\text { de sensibilisation aux NTIC }\end{array}$ \\
\hline $\begin{array}{l}\text { Appel à projets lancé par le } \\
\text { Réseau national de recherche } \\
\text { en télécommunications - RNRT }\end{array}$ & $\begin{array}{l}04 / 1998- \\
09 / 1998\end{array}$ & $\begin{array}{l}\text { Réseau national de recherche } \\
\text { en télécommunications }\end{array}$ & $\begin{array}{l}\text { Favoriser le transfert technologique } \\
\text { et le dialogue entre la recherche } \\
\text { en amont et la recherche et } \\
\text { développement appliquée }\end{array}$ \\
\hline $\begin{array}{l}\text { Appel à projets "Utilisation } \\
\text { collective d'Internet par } \\
\text { les PMI" (hors BTP, IAA } \\
\text { et transports) }\end{array}$ & $\begin{array}{l}05 / 1998- \\
09 / 1998\end{array}$ & Secrétariat d'État à l'Industrie & $\begin{array}{l}\text { Encourager les initiatives collectives et } \\
\text { innovantes destinées à aider les PME-PMI } \\
\text { à s'approprier les technologies Internet }\end{array}$ \\
\hline $\begin{array}{l}\text { Appel à projets "Offre } \\
\text { de procédés et produits de } \\
\text { sécurisation pour la mise en } \\
\text { oeuvre des autoroutes de } \\
\text { l'information" (OPPIDUM) }\end{array}$ & $\begin{array}{l}09 / 1998- \\
01 / 1999\end{array}$ & $\begin{array}{l}\text { Service des industries de } \\
\text { communication et de service } \\
\text { du Secrétariat à l'industrie, et } \\
\text { Service central de la sécurité } \\
\text { des systèmes d'information }\end{array}$ & $\begin{array}{l}\text { Favoriser le développement du commerce } \\
\text { électronique, la première concerne } \\
\text { la mise en œuvre } \\
\text { de la politique de sécurité }\end{array}$ \\
\hline $\begin{array}{l}\text { Appel à projets pour le } \\
\text { développement de la société } \\
\text { de l'information dans la } \\
\text { Région Nord - Pas de Calais }\end{array}$ & & $\begin{array}{l}\text { Conseil régional Nord - } \\
\text { Pas de Calais, préfecture } \\
\text { de Région et Commission } \\
\text { européenne }\end{array}$ & $\begin{array}{l}\text { Développer de nouveaux services liés à } \\
\text { l'emploi, à la formation continue, aux } \\
\text { nouvelles formes d'organisation } \\
\text { du travail; service pour les PME-PMI }\end{array}$ \\
\hline $\begin{array}{l}\text { Appel à propositions } \\
\text { sur les autoroutes et services } \\
\text { de l'information }\end{array}$ & $\begin{array}{l}\text { Perma- } \\
\text { nent }\end{array}$ & Secrétariat d'État à l'Industrie & $\begin{array}{l}\text { Promotion de nouveaux services, et mise } \\
\text { en place des plates-formes de } \\
\text { développement avec réseaux à hauts débits }\end{array}$ \\
\hline $\begin{array}{l}\text { Appel d'offres et } \\
\text { labellisation "Exportateurs } \\
\text { sur la Toile" }\end{array}$ & $\begin{array}{l}07 / 1998- \\
07 / 2000\end{array}$ & $\begin{array}{l}\text { Secrétariat d'État au } \\
\text { Commerce extérieur }\end{array}$ & $\begin{array}{l}\text { Encourager les entreprises à utiliser } \\
\text { les NTIC et particulièrement Internet } \\
\text { pour développer leur présence } \\
\text { à l'international }\end{array}$ \\
\hline Appel à projets "Perin@t" & $\begin{array}{l}01 / 1999- \\
04 / 1999\end{array}$ & $\begin{array}{l}\text { Fonds national } \\
\text { d'aménagement et de } \\
\text { développement du territoire }\end{array}$ & $\begin{array}{l}\text { Financer des projets de réseaux reliant } \\
\text { des maternités et des centres périnataux }\end{array}$ \\
\hline $\begin{array}{l}\text { Appel à projets Multimédia } \\
\text { en région Languedoc- } \\
\text { Roussillon }\end{array}$ & $\begin{array}{l}10 / 1998- \\
12 / 1999\end{array}$ & $\begin{array}{l}\text { Direction Régionale de } \\
\text { l'Industrie, de la recherche } \\
\text { et de l'environnement et } \\
\text { Conseil Régional } \\
\text { Languedoc-Roussillon }\end{array}$ & $\begin{array}{l}\text { Faciliter l'accès à des financements pour } \\
\text { les entreprises de la région, de moins de } \\
500 \text { personnes, participant à l'industrie } \\
\text { du multimédia }\end{array}$ \\
\hline $\begin{array}{l}\text { Appel à projets } \\
\text { d'expérimentation de } \\
\text { nouveaux services techniques } \\
\text { ou de projets d'innovation } \\
\text { technologiques }\end{array}$ & $\begin{array}{l}\text { Perma- } \\
\text { nent }\end{array}$ & $\begin{array}{l}\text { Service des Technologies et } \\
\text { de la société de l'information } \\
\text { de la Direction générale de } \\
\text { l'Industrie, des technologies } \\
\text { de l'information et des postes }\end{array}$ & $\begin{array}{l}\text { Favoriser le développement de nouveaux } \\
\text { services tels que : la télémédecine, } \\
\text { la formation à distance, la mise } \\
\text { en place d'un porte-monnaie } \\
\text { électronique... }\end{array}$ \\
\hline
\end{tabular}

